\title{
AN APPLICATION OF THE MOORE-PENROSE INVERSE TO ANTISYMMETRIC RELATIONS
}

\author{
ROBERT E. HARTWIG
}

\begin{abstract}
Let $\boldsymbol{R}$ be a star-ring and let $\boldsymbol{R}_{\boldsymbol{\dagger}}$ denote the set of star-regular elements in $R$. It is shown that the relation $a \Delta b$, defined by $a a^{*} a=a b^{*} a$, is antisymmetric on $\boldsymbol{R}_{\boldsymbol{t}}$ provided that the two-term star-cancellation law and the positive-semidefinite axiom hold in $R$. This includes the star-regular elements of all $C^{*}$-algebras, and in particular those elements in $\mathbf{C}_{n \times n}$ and $B(H)$, the bounded linear transformations on Hilbert space $H$.
\end{abstract}

1. Introduction. One of the most striking results in the recent study of generalized inverses, is the result by Rao, Mitra and Bhimasankaram [8], which states that for complex $m \times n$ matrices, $A$ and $B$,

$$
A B^{\dagger} A=A, B A^{\dagger} B=B \Rightarrow A=B,
$$

in which $(\cdot)^{\dagger}$ denotes the Moore-Penrose inverse of the matrix $(\cdot)[1]$. The proof given in [8] was based on the singular value decomposition theory, which is essentially finite dimensional and nonalgebraic in nature.

The purpose of this paper is to extract the purely algebraic conditions, which make this result valid, and to extend the class of objects for which (1) remains true.

We shall state our results in terms of ring elements, from which the specific applications to (rectangular) matrices and operators easily follow.

Let $R$ be a*-ring (that is a ring with involution $\left.(\cdot)^{*}\right)[2$, p. 1]. We say that the $k$-term star-cancellation law holds, if

$$
\left(\mathrm{SC}_{k}\right) \quad a_{1}^{*} a_{1}+\cdots+a_{k}^{*} a_{k}=0 \Rightarrow a_{1}=a_{2}=\cdots=a_{k}=0 \text {. }
$$

The involution is called proper if $\mathrm{SC}_{1}$ holds. Throughout this paper we shall assume at most a two-term global star-cancellation law. In addition we shall need the positive-semidefinite axiom:

$$
\text { (P.S.D.) } a a^{*} b b^{*}=b b^{*} a a^{*} \Rightarrow a a^{*} b b^{*}=c c^{*} \text {, for some } c \in R \text {. }
$$

This axiom generalizes the well-known result that for projections in a proper *-ring

$$
e f=f e \Leftrightarrow(e f)^{2}=e f(e f)^{*} \Leftrightarrow(e f)^{2}=e f .
$$

It will be shown that on $R_{\dagger}=\{a \mid a$ is star-regular $\}$, the relations

$$
a \triangle b \quad \text { iff } a a^{*} a=a b^{*} a,
$$

Received by the editors March 7, 1978 and, in revised form, November 22, 1978.

AMS (MOS) subject classifications (1970). Primary 16A28, 16A30; Secondary 15A09. 
and

$$
a \square b \text { iff } a=a b^{\dagger} a,
$$

are both antisymmetric as well as reflexive. This includes the corresponding results for $C^{*}$-algebras with unity [2], [7, p. 33], such as $\mathbf{C}_{n \times n}$ and $B(H)$, the bounded linear operators on Hilbert space. It will further become apparent that the antisymmetry of $\triangle$ and $\square$ is a consequence of a delicate interaction between the $\mathrm{SC}_{2}$ axiom and the star-orthogonal partial ordering [3, Theorem 6.3],

$$
p \leqslant q \Leftrightarrow p^{*}(p-q)=0=(p-q) p^{*}
$$

Let us begin by defining our main concepts.

An element $a \in R$ is called regular if $a \in a R a$ and, *-regular if its Moore-Penrose inverse $a^{\dagger}$ exists. That is, if there is a (necessarily unique) solution to the equations

$$
a x a=a, \quad x a x=x, \quad(a x)^{*}=a x, \quad(x a)^{*}=x a .
$$

It is well known [9], that locally the existence of $a^{\dagger}$ is equivalent to the regularity of $a a^{*}$ and $a^{*} a$, combined with the existence of the local starcancellation laws

$$
\left(a^{*} a\right)^{0}=a^{0}, \quad{ }^{0}\left(a^{*} a\right)={ }^{0}\left(a^{*}\right)
$$

where $a^{0}=\{x \in R \mid a x=0\},{ }^{0} a=\{x \in R \mid x a=0\}$. A ring is called (star-) regular when every element $a$ in $R$ is (star-) regular. Star-regularity occurs precisely when globally the involution is proper and $a^{*} a$ is regular. A *-ring with unity is symmetric [2, p.9], if $\left(1+a^{*} a\right)$ is a unit for all $a \in R$. An element $a \in R$ belongs to a multiplicative subgroup if and only if there is a solution to the equations [6, p. 205],

$$
a x a=a, \quad x a x=x, \quad a x=x a .
$$

This solution is necessarily unique and is called the group inverse $a^{\#}$ of $a$ [5].

2. Preliminary results. We shall start by deriving some local equivalent formulations to (1) which do not need the $\mathrm{SC}_{2}$ or P.S.D. axioms.

THEOREM 1. Let $a$ and $b$ be star-regular elements in a star-ring $R$. Then

( $\alpha) a \square b \Leftrightarrow a^{\dagger} \triangle b^{\dagger}$,

( $\beta$ ) $\square$ is antisymmetric if and only if $\triangle$ is antisymmetric.

In addition the following are equivalent:

(i) $a \square b$ and $b \square a$,

(ii) $a^{\dagger} \square b^{\dagger}$ and $b^{\dagger} \triangle a^{\dagger}$,

(iii) $a \triangle b$ and $b \triangle a$,

(iv) $a^{\dagger} \square b^{\dagger}$ and $b^{\dagger} \square a^{\dagger}$.

In which case

(v) $\left(a b^{\dagger}\right)^{*}=b a^{\dagger},\left(b^{\dagger} a\right)^{*}=a^{\dagger} b$. 
Proof. ( $\alpha$ ) Let $a \square b$ or $a b^{\dagger} a=a$. Then $a a^{\dagger}=a b^{\dagger} a a^{\dagger}=\left(a b^{\dagger} a a^{\dagger}\right)^{*}=$ $a a^{\dagger} b^{* \dagger} a^{*}$ and thus $a^{\dagger}=a^{\dagger} b^{* \dagger} a^{*}$. Now $\left(a a^{*} a\right)^{\dagger}=a^{\dagger} a^{* \dagger} a^{\dagger}=a^{\dagger} b^{* \dagger} a^{\dagger}$ and so $a^{\dagger} \triangle b^{\dagger}$. Each step is clearly reversible. Moreover, if $b \square a$, then $b a^{\dagger}=$ $b\left(a^{\dagger} b^{* \dagger} a^{*}\right)=\left(b a^{\dagger} b\right) b^{\dagger} b^{* \dagger} a^{*}=b b^{\dagger} b^{* \dagger} a^{*}=\left(a b^{\dagger}\right)^{*}$.

$(\beta)$ This follows at one from part $(\alpha)$ and the uniqueness of $(\cdot)^{\dagger}$.

Now consider the remaining conditions. From $(\alpha)$ it is clear that (i) $\Leftrightarrow$ (ii) and (iii) $\Leftrightarrow$ (iv). So suppose that (i) holds. Then as in ( $\alpha), a^{\dagger}=a^{\dagger} b^{* \dagger} a^{*}$ and hence $b b^{*} b=b b^{*} b a^{\dagger} b=b b^{*} b a^{\dagger} b^{* \dagger} a^{*} b=b b^{*}\left(b a^{\dagger} b\right) b^{\dagger} b^{* \dagger} a^{*} b=$ $b b^{*} b b^{\dagger} b^{* \dagger} a^{*} b=b a^{*} b$, or $b \triangle a$. Symmetry now also yields $a \triangle b$, and (iii) holds. Next, replacing $a$ by $a^{\dagger}, b$ by $b^{\dagger}$ shows that (iv) $\Rightarrow$ (ii), completirg the equivalence. The first identity of $(v)$ was proven in $(\alpha)$, while the remaining identity again follows by symmetry. This completes the proof.

It should be remarked here, that the individual parts of each of the four equivalent conditions do not yield as much information as the combined conditions do. For example, $a \square b \Rightarrow a \triangle b$, yet 9(i) $\Rightarrow$ 9(iii).

Because the conditions of Theorem 1 do not seem to suffice for the antisymmetry of $\Delta$, let us now impose two realistic global assumptions of $R$ which will ensure this fact.

\section{Main results.}

TheOREM 2. Let $R$ be $a^{*}$-ring, and let $a, b$ be star-regular elements of $R$. Assume further that the two-term star-cancellation law $S C_{2}$, as well as the P.S.D. axiom hold globally in $R$. Then $\triangle$ and $\square$ are both antisymmetric.

Proof. Suppose $a b^{\dagger} a=a$ and $b a^{\dagger} b=b$. Now consider the identity:

$$
a b^{\dagger}\left(1-a a^{\dagger}\right) b a^{\dagger}+a\left(1-b^{\dagger} b\right) a^{\dagger} \equiv 0,
$$

and set $p=a b^{\dagger}\left(1-a a^{\dagger}\right), q=a\left(1-b^{\dagger} b\right)$. Since $b a^{\dagger}=\left(a b^{\dagger}\right)^{*}$ we have $p p^{*}+$ $a\left(1-b^{\dagger} b\right) a^{\dagger}=0$, or

$$
p p^{*} a a^{*}+a\left(1-b^{\dagger} b\right) a^{*}=p p^{*} a a^{*}+q q^{*}=0 .
$$

Starring this shows that $p p^{*} a a^{*}=a a^{*} p p^{*}$, and hence by the P.S.D. axiom (3), $p p^{*} a a^{*}=c c^{*}$ for some $c$. Consequently

$$
c c^{*}+q q^{*}=0
$$

which by $\mathrm{SC}_{2}$ implies that $c=q=0$. Now $q=0 \Rightarrow a=a b^{\dagger} b \Rightarrow b^{\dagger} a=b^{\dagger} b$, while

$$
c=0 \Rightarrow 0=p^{*} a=\left(1-a a^{\dagger}\right) b a^{\dagger} a \Rightarrow b a^{\dagger} a=a a^{\dagger} b a^{\dagger} a=a \Rightarrow b a^{\dagger}=a a^{\dagger} .
$$

Interchanging $a$ with $b$ now yields the four equations

$$
\begin{array}{ll}
a^{\dagger} b=a^{\dagger} a, & b^{\dagger} a=b^{\dagger} b \\
b a^{\dagger}=a a^{\dagger}, & a b^{\dagger}=b b^{\dagger} .
\end{array}
$$


Hence $b=\left(b a^{\dagger}\right) b=a\left(a^{\dagger} b\right)=a a^{\dagger} a=a$, as desired. It should be pointed out here that the equations (13) precisely state that $a \preccurlyeq b$ and $b \preccurlyeq a$, [3, Corollary 6.6].

COROLlaRY. If $R$ is a star-ring with global $S C_{2}$ and P.S.D. axioms, then the relations $\triangle$ and $\square$ are antisymmetric on $R_{\dagger}=\left\{a \mid a^{\dagger}\right.$ exists $\}$.

Let us conclude with several remarks and close with some open problems.

4. Conclusions and remarks. The P.S.D. axiom (3) is weaker than the weak square root axiom (WSR) [2, p. 66], which assumes that for all $a \in R$, there is $r \in R$ such that

$$
a a^{*}=r r^{*}=r^{*} r, \quad a a^{*} q=q a a^{*} \Rightarrow r q=q r .
$$

Indeed, if $a a^{*} b b^{*}=b b^{*} a a^{*}$, then $r b b^{*}=b b^{*} r \Rightarrow r^{*} b b^{*}=b b^{*} r^{*}$ and so $a a^{*} b b^{*}=r r^{*} b b^{*}=r b b^{*} r^{*}=c c^{*}$.

It is not known whether the converse is true. The WSR axiom is known to hold in all $C^{*}$-algebras with unity [7, p. 33], and in particular in $\mathbf{C}_{n \times n}$ and $B(H)$.

We conjecture that the relation $a \triangle b: a a^{*} a=a b^{*} a$ is antisymmetric on the entire star-ring, provided that the $\mathrm{SC}_{2}$ and P.S.D. axioms hold. Three pertinent observations in this direction are that in a proper *-ring,

(i) $\triangle$ is antisymmetric on projections.

(ii) If $a \Delta b$ and $b \triangle a$, then $a$ is a partial isometry if and only if $b$ is a partial isometry.

(iii) If $a \triangle b$ and $b \triangle a$ then $a^{\dagger}$ exists if and only if $b^{\dagger}$ exists.

The results of Theorem 1 are easily seen to hold in a ${ }^{*}$-semigroup [3], while the results of Theorem 2 can be modified without difficulty to include matrices or bounded linear maps from one Hilbert space into another. It is not known how much, if any, of Theorem 2 carries over to *-semigroups.

Indeed, in order to extend Theorem 2 to $R_{m \times n}$, the set of $m \times n$ matrices over $R$, with, say $m \leqslant n$, it suffices to consider $R_{n \times n}$ since we may always add zeros, and $\left[\begin{array}{l}A \\ 0\end{array}\right]^{\dagger}=\left[A^{\dagger}, 0\right]$, if any. In order to define $A^{\dagger}$ for $A \in R_{n \times n}$, we first need an involution on $R_{n \times n}$. This may be defined in the usual way by setting $\left(A^{*}\right)_{i j}=a_{j i}^{*}$. Using this involution it is now easily seen that $\mathrm{SC}_{k}$ holds in $R_{n \times n}$ exactly when $\mathrm{SC}_{k n}$ holds in $R, k=1,2, \ldots$, which underlines one of the severe shortcomings of this particular involution. We may similarly state the P.S.D. axiom for $R_{n \times n}$, which must imply the same axiom for $R$, but not conversely. The proof of Theorem 2 , now carries over verbatim to $R_{n \times n}$.

We may subsequently say that it is of some interest to investigate the relations, if any, between the various star-cancellation laws. The following is a first step in this direction.

The one and two-term star-cancellation laws are not entirely unrelated. The concept needed to connect them is that of symmetry. Globally we have the following result. 
TheOREM 3. Let $R$ be $a^{*}$-ring with unity. Then the following are equivalent in sets

(a) (i) $a^{*} a+b^{*} b$ is regular for all $a, b \in R$,

(ii) $S C_{2}$ holds,

( $\beta$ ) (i) $a^{*} a+1$ is regular for all $a \in R$,

(ii) $a^{*} a$ is regular for all $a \in R$,

(iii) $S C_{2}$ holds,

( $\gamma) \quad(i) \quad R$ is symmetric,

(ii) $a^{*} a$ is regular for all $a$ in $R$,

(iii) $S C_{1}$ holds,

( $\delta$ ) (i) $R$ is symmetric,

(ii) $R$ is regular,

In which case $R$ is ${ }^{*}$-regular.

Proof. $(\alpha) \Rightarrow(\beta)$ Clear.

$(\beta) \Rightarrow(\gamma)$. All that is required is to prove that $R$ is symmetric. Now let $\left(1+a^{*} a\right) x=0$. Then $x^{*} x+(a x)^{*} a x=0$ and hence by $\mathrm{SC}_{2}, x=0$. This means that $\left[R\left(1+a^{*} a\right)\right]^{0}=(0)$, and because $\left(1+a^{*} a\right)$ is regular, $R(1+$ $\left.a^{*} a\right)={ }^{0}\left(\left[R\left(1+a^{*} a\right)\right]^{0}\right)=R$. Similarly $\left(1+a^{*} a\right) R=R$, ensuring that $1+$ $a^{*} a$ is a unit.

$(\gamma) \Rightarrow(\beta)$. We begin by noting that $(\gamma)$ (ii) together with $(\gamma)($ iii), are equivalent to the star-regularity of $R$. Now suppose that $a^{*} a+b^{*} b=0$. Then

$$
a^{* \dagger} b^{*} b a^{\dagger}+a a^{\dagger}=0 \text { and } 1+\left(b a^{\dagger}\right)^{*} b a^{\dagger}=1-a a^{\dagger} .
$$

Since $R$ is symmetric, both sides must be a unit, which forces $a a^{\dagger}=0$. Hence $a=0$ and $b=0$.

$(\gamma) \Rightarrow(\delta)$. Obvious since $R$ is star-regular.

$(\delta) \Rightarrow(\gamma)$. If $R$ is regular, then for any $a \in R, a R=e R, R a=R f$ for some idempotents $e, f$. If $R$ is symmetric, then it follows by a result of Kaplansky [7, p. 34], that $e R=e_{1} R, R f=R f_{1}$, for some projections $e_{1}, f_{1}$ in $R$. Hence $a^{\dagger}$ exists [6, p. 202], and $(\gamma)$ follows.

$(\beta) \Rightarrow(\alpha)$. Clear since $(\beta)$ ensures that $R$ is star-regular.

Related to the P.S.D. and $\mathrm{SC}_{3}$ axioms is the following reduction law:

$$
\left(1+a a^{*}\right)\left(1+b b^{*}\right)=1 \Rightarrow a=b=0,
$$

which actually is implied by $\mathrm{SC}_{3}$. In fact,

$$
\begin{aligned}
-b b^{*} & =1-\left(1+b b^{*}\right)=1-\left(1+a a^{*}\right)^{-1}=a\left(1+a^{*} a\right)^{-1} a \\
& =a\left(1+a^{*} a\right)^{-2} a^{*}+a\left(1+a^{*} a\right)^{-1} a^{*} a\left(1+a^{*} a\right)^{-1} a,
\end{aligned}
$$

which by $\mathrm{SC}_{3}$ forces $a=b=0$. It is not known whether the $\mathrm{SC}_{3}$ and P.S.D. axioms suffice for (16) to hold. 
In conclusion, let us remark that neither $\Delta$ nor $\square$ will be transitive in

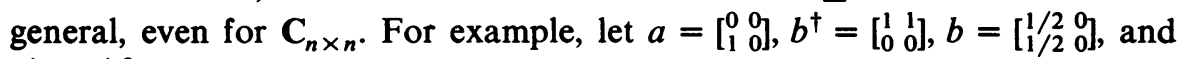
$c^{\dagger}=\left[\begin{array}{ll}4 & 0 \\ 0 & 4\end{array}\right]$. No suitable extra conditions are known that will make $\Delta$ or transitive. Lastly, in a *-ring with P.S.D. axiom, does algebraic equivalence of projections imply star-equivalence $[2$, p. 66]?

\section{REFERENCES}

1. A. Ben Israel and T. N. E. Greville, Generalized inverses, theory and applications, Wiley, New York, 1974.

2. S. K. Berberian, Baer star-rings, Springer-Verlag, Berlin and New York, 1972.

3. M. P. Drazin, Natural structures on rings and semigroups with involution (submitted for publication).

4.

, Pseudo inverses in associate rings and semigroups, Amer. Math. Monthly 65 (1958), 506-514.

5. I. Erdelyi, On the matrix equation $A x=\lambda B x$, J. Math. Anal. Appl. 17 (1967), 119-132.

6. R. E. Hartwig, Block generalized inverses, Arch. Rational Mech. Anal. 61 (1976), 197-251.

7. I. Kaplansky, Rings of operators, Benjamin, New York, 1968.

8. C. D. Rao, S. K. Mitra and P. Bhimasankaram, Determination of a matrix by its subclasses of generalized inverses, Sankhyā Ser. A 34 (1972), 5-8.

9. N. S. Urquhart, Computation of generalized inverse matrices which satisfy specified conditions, SIAM Rev. 10 (1968), 216-218.

Department of Mathematics, North Carolina State University, Raleigh, North CAROLINA 27607 